\title{
The Effect of Texturing on the Efficiency of a-Si Solar Cell
}

\author{
Bassam M. Mustafa \\ Department of Physics \\ College of Science \\ Mosul University
}

(Received 15/ 1/2009; Accepted 13/4/2009)

\begin{abstract}
Texturing of amorphous silicon( a-Si ) solar cell surface by parallel grooves leads to collecting the reflected light from one side of the groove by the other side. In this work a theoretical method is found to calculate the effect of texturing on the efficiency of the $(\mathrm{a}-\mathrm{Si})$ solar cells. This is done by finding the change in the photo current due to the increase of light energy trapped due to texturing. Then new I-V are found for groove angles (acute, right and obtuse angles) and for angles of incidence from ( 0 up to $180^{\circ}$ ). This is done by finding the solar cell parameters (photo current, total current, short circuit current, open circuit voltage). For small groove acute angels ( 40 as example ) about $100 \%$ collection of the incident energy is happened, this in turn increases the efficiency, but this happen within limited angular range. Increasing the groove angles increases the range of maximum absorption but lowers the efficiency.
\end{abstract}

Keywords : a-Si Solar cells, texturing of solar cells, texturing by parallel grooves

\section{تأثير الثثكل علل كماءة خلية للسيكهن غير المتبلور الثهسية}

\section{ll}

تشكل سطح الخلية اللشمسية السيليكونية غير المتبلورة بلأخاديد طولية يودي إلى تجمبع الأشعة المنعكسة من طرف الأخدود بولنطة الطرف الآخر. في هذا البهث وجدت طبريقة لهسلب تأثيدر التشكيل

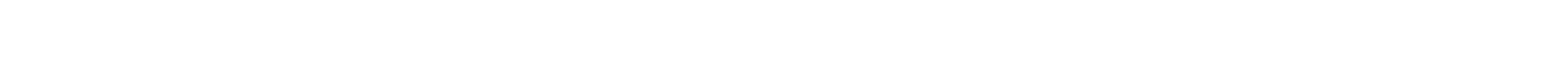

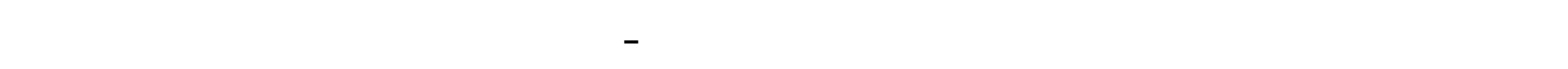

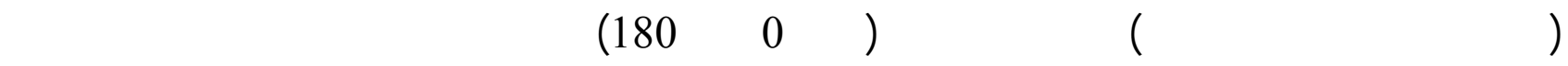

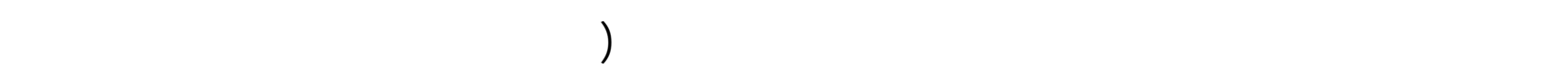

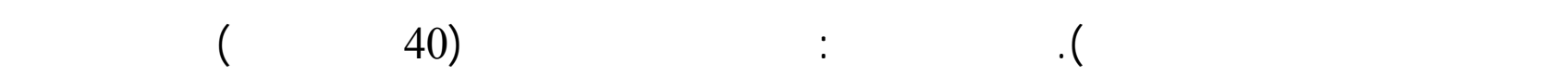

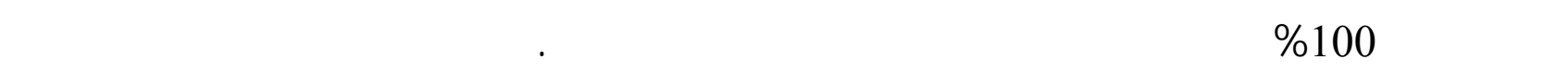
الزاوي ولكنه يقص الكفاءة . 


\section{INTRODUCTION}

Thin film polycrystalline efficient solar cells conversion requires that the photovoltaic materials are capable of absorbing a significant fraction of energy from sunlight (Dragoman, 2002). A good performance a-Si: H solar cells are usually made in $p-i-n$ configuration since 1980 (Carlson, 1984 ). The first type of this solar cells is from amorphous silicon (Nelson, 2003). Soon great attention was given for this type of solar cells for many reasons, like the easy way of fabrication of these cells, and as a result the cheap price of these cells in comparison with the single crystalline solar cells. The first generations of these solar cells was of low efficiency, but soon great efforts were done which lead quickly to improve the efficiency, such that it reach up to the limit of 10\% (green, 1995). Direct texturing of silicon, as used for light - trapping and reflection control with wafer cells, could be difficult to apply for thin films. An alternative method is to texture the substrate, directly or by prior deposition of a textured dielectric or transparent conducting oxide especially for a-Si: H solar cells (Thorp et al.,1997). Macroscopic geometric texture provide a microscopically smooth surface and could be formed at low cost by technique like embossing. This was investigated for a-Si: $\mathrm{H}$ thin film solar cells (Brendel, 1996). By texturing of feature sizes greater than the thickness of the film the front surface of the silicon conforms to the texture of the substrate, thus retaining the texture anti-reflection properties (Thorp et al., 1997 ).

. In this research a study of the effect of surface texturing on trapping light on the efficiency of amorphous silicon (a-Si ) solar cells is to be done, for this reason, a method to calculate the generated photo - current must be done. This current is to be used to find the $\mathrm{I}-\mathrm{V}$ characteristics and from it, the fill factor and efficiency can be found for the cell without texturing. Then the effect of texturing on the absorbed light has to be found and the final efficiency for texturing with parallel grooves is to be found.

\section{THEORETICAL PART}

A high performance a-Si: $\mathrm{H}$ solar cells $(\eta>7 \%)$ have been fabricated in many different structures. One of the highest performance $(\eta=10 \%)$ have the structure glass $/ \mathrm{SnO}_{2} / p-i-n / \mathrm{Ag}$ in which the p layer is an alloy of a-Si: C : H (Swartz, 1984 ) as in fig. 1.
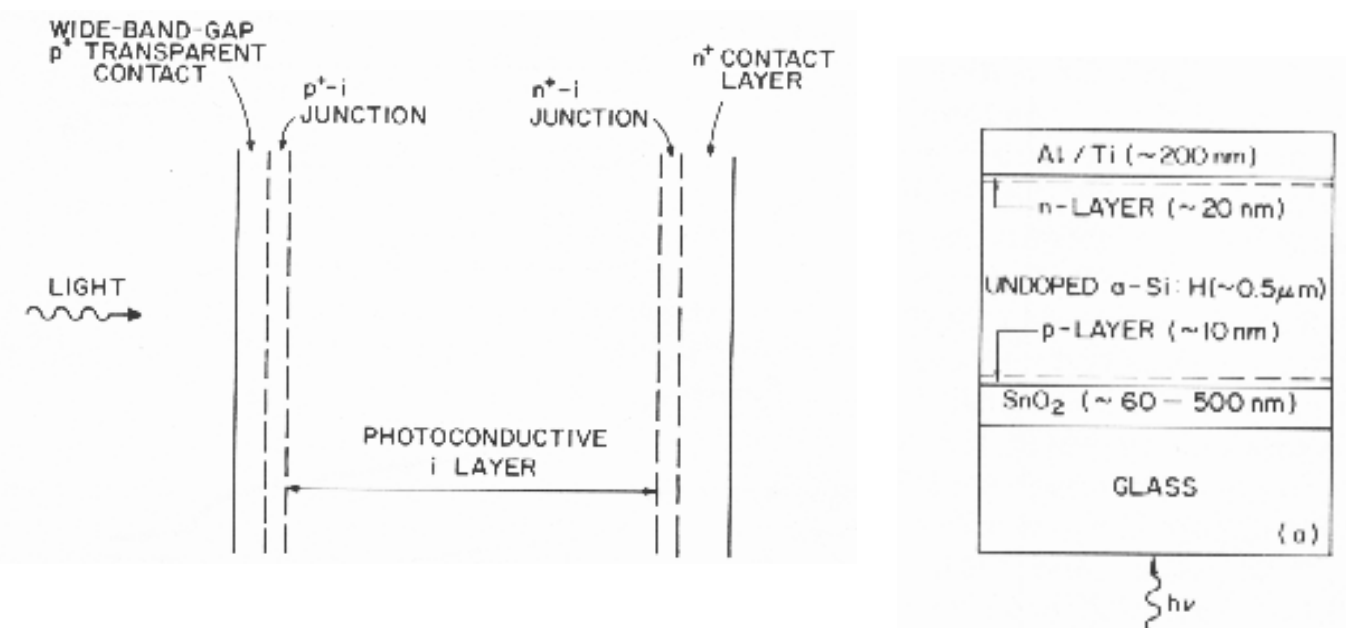

Fig.1: (left ) highest performance $\left(\eta=10 \%\right.$ ) have the structure glass $/ \mathrm{SnO}_{2} / p-i-n / \mathrm{Ag}$ . ( Swartz, 1984). (right ) model for the former layer 
Generally this cell has optical gap $\sim 2 \mathrm{eV}$ (Swartz, 1984 ) the thickness of the $\mathrm{SnO}_{2}$ layer so chosen to act as antireflection coating, or it may be thicker to have sheet resistance of the order $\sim(1-10) \Omega / \mathrm{cm}^{2}$.

The principle method of characterizing solar- cell performance is the measurement of conversion efficiency while the cell is exposed to one sun illumination $\left(\sim 100 \mathrm{~m} \mathrm{~W} / \mathrm{cm}^{2}\right)$. The conversion efficiency is determined by measuring the $I-V$ characteristics, locating the maximum power point $\left(P_{m}=J_{m} V_{m}\right)$ and the solar insolation $\left(P_{i}\right)$, then the conversion efficiency :

$$
\eta=\frac{J_{m} V_{m}}{P_{i}}
$$

It is worth noting that the performance characteristics of a variety of cells is different ( Carlson, 1984 ).

There have been several models of operation of a-Si: H solar cells starting from Poisson equation and the continuity equations for electron and holes ( Swarts, 1982; Crandall, 1982) and other models, all these models produced results that are in reasonable agreement with the $I-V$ characteristics of a-Si: H solar cells. From the energy band diagram for the $p-i-n$ structure (Crandall, 1982) found that the photo current density can be written

$$
J=q G l_{c}\left[1-\exp \left(-d / l_{c}\right)\right]
$$

where $G$ is the generation rate of the electron hole pairs and, $d$ is the thickness of the $i$ layer and $l_{c}$ is the sum of the electron and hole drift lengths (Faughnan et al.,1984) which can be written as :

$$
l_{c}=\mu_{n} \tau_{n} \varepsilon+\mu_{p} \tau_{p} \varepsilon
$$

The generation rate $G$ of the electron hole pairs is

$$
G(\lambda)=\alpha(\lambda) N(\lambda)[1-R(\lambda)] \exp (-\alpha(\lambda) x)
$$

where $\alpha(\lambda), N(\lambda), R(\lambda)$ are the absorption coefficient, the photon current and the reflection coefficient respectively for certain wave length .

The proposed model for a-Si: H solar cells as shown in the right side of fig. 1 consist of two junctions interconnected by the photoconductive $i$ layer where the length of this region is much greater than the lengths of the interface layers. The light enters the cell through the $\mathrm{P}^{+}$layer and the sign convention is such that the short -circuit current is positive. The total current density $J_{T}$ through the cell is :

$$
J_{T}=J_{p}-J_{d}
$$

where $J_{d}$ is the diffusion current density, which can be written as :

$$
J_{d}=n q l / \tau_{n}
$$

where $n, q, \tau_{n}$, are the density, charge, and the relaxation time for the electrons in the I layer. Then the I-V can be described by the following equation (Swartz, 1984)

$V=-R_{c} J_{T}-\frac{V_{s}\left(1-0.5 \sqrt{\left.J_{T} / J_{p}\right)}\right.}{J_{p} / J_{T}-1}-\frac{\beta k T}{q} \ln \left(1-\frac{J_{T}}{J_{p}}\right)^{-1}+V_{\text {oc }} \ldots \ldots$

This equation to a good extent coincided with the experimental results then the efficiency can determined by determining $J_{m}$ and $V_{m}$. 


\section{The effect of texturing}

In order to calculate the increase in efficiency of the a-Si solar cells we try to increase its ability of gathering the lost rays by reflection from the surface of the solar cell, here a study of the effect of texturing by parallel grooves on a substrate is done. Then the solar cell will matches the texturing of the substrate as shown in fig. 2 , as we can see the grooves are greater than the solar cell thickness .

The grooves will catch escaped rays from one side by the other. About $80 \%$ of the escaped energy in the first reflection can be gathered This as a result increases the photo current which comes from increasing of the generation rate .

In order to find the photo current from 7 we do the following:

In case of short circuit case

$$
V=0
$$

$0=-R_{c} J_{T}-\frac{V_{s}\left(1-0.5 \sqrt{\left.J_{T} / J_{p}\right)}\right.}{J_{p} / J_{T}-1}-\frac{\beta k T}{q} \ln \left(1-\frac{J_{T}}{J_{p}}\right)^{-1}+V_{o c}$

Rearrange the former equation

$R_{c} J_{T}+\frac{V_{s}\left(1-0.5 \sqrt{\left.J_{T} / J_{p}\right)}\right.}{J_{p} / J_{T}-1}=\frac{\beta k T}{q} \ln \left(1-\frac{J_{T}}{J_{p}}\right)+V_{o c}$

Now to solve for $J_{T}$ from $\ln \left(1-\frac{J_{T}}{J_{P}}\right)$ we have

$\frac{J_{T}}{J_{P}} \cong 1 \quad$ therefore

$\ln (1-x)=-x-\frac{x^{2}}{2}-\frac{x^{3}}{3}+\ldots \ldots \ldots$.

$\ln \left(1-\frac{J_{T}}{J_{P}}\right) \cong-\frac{J_{T}}{J_{P}}-\frac{1}{2}-\frac{1}{3}$

$R_{c} J_{T}+\frac{V_{s}\left(1-0.5 \sqrt{\left.J_{T} / J_{p}\right)}\right.}{J_{p} / J_{T}-1}=\frac{\beta k T}{q}\left(-\frac{J_{T}}{J_{P}}-\frac{1}{2}-\frac{1}{3}\right)+V_{o c}$

Since $\sqrt{\frac{J_{T}}{J_{p}}} \cong 1 \quad:$ then 


$$
\begin{aligned}
& R_{c} J_{T}+\frac{0.5 V_{s}}{J_{p} / J_{T}-1}=\frac{\beta k T}{q}\left(-\frac{J_{T}}{J_{P}}-\frac{1}{2}-\frac{1}{3}\right)+V_{o c} \\
& \text { let } \frac{\beta k T}{q}=c_{1} \quad \text { and } \quad \frac{J_{T}}{J P}=c_{2} \\
& R_{c} J_{T}+\frac{0.5 V_{s}}{1 / c_{2}-1}=c_{1}\left(-c_{2}-\frac{1}{2}-\frac{1}{3}\right)+V_{o c} \\
& c_{1} c_{2}^{2}+\left[-\frac{1}{6} C_{1}+V_{o c}-0.5 V_{s}+R_{c} J_{T}\right] c_{2}+V_{o c}-R_{c} J_{T}-\frac{5}{6} C_{1}=0
\end{aligned}
$$

equation 15 is a second order equation and can : be solved as follows

$$
c_{2}=\frac{-B \pm \sqrt{B^{2}-4 A C}}{2 A}
$$

where $A=c_{1} \quad: B=\left[-\frac{1}{6} c_{1}+V_{o c}-0.5 V_{s}+R_{c} J_{T}\right]: C=V_{o c}-R_{c} J_{T}-\frac{5}{6} c_{1}$ substituting for $V_{o c}, V_{s}, R_{c}, \beta, k, q, T$ we can find the value of $J_{T}$ for any value of $J_{p}$.

After entering the effect of texturing the following must be done :

By joining equations 2 and 4 the photo current is :

$$
J_{p}=q l_{c} \alpha(\lambda) N(\lambda)[1-R(\lambda)]\left(\exp (-\alpha(\lambda) x)\left[1-\exp \left(-d / l_{c}\right)\right]\right.
$$

The effect of texturing comes mainly from the change of the of $R(\lambda)$ (decrease of reflection coefficient as a result of texturing ). Then equation 17 becomes :

$$
J_{p}=c_{3}[1-R(\lambda)]
$$

where

$c_{3}=q l_{c} \alpha(\lambda) N(\lambda)\left(\exp (-\alpha(\lambda) x)\left[1-\exp \left(-d / l_{c}\right)\right]\right.$

Therefore if the $J_{P 1}, R_{1}$ are the photo current and the reflection coefficient before texturing and $J_{P 2}, R_{2}$ are their values after texturing then 
$J_{p 2} / J_{p 1}=\left[1-R_{2}\right] /\left[1-R_{1}\right]$

To find the effect of texturing on the open circuit voltage, for the a-Si solar cells the open circuit voltage $V_{o c}$ and the short circuit current density $J_{s c}$ are related by the following equation

$V_{o c}=(k T / q) \ln \left(J_{s c} / J_{o}\right)+1$

In order to calculate the change in the open circuit voltage by change in the absorbed light, let $V_{o c 1}$ represent the initial case and $V_{o c 2}$ represent the case after additional light is absorbed due to the effect of texturing, then for the first case

$$
V_{o c 1}=(k T / q) \ln \left(J_{s c 1} / J_{o}\right)+1
$$

and for the second case

$$
V_{\text {oc } 2}=(k T / q) \ln \left(J_{s c 2} / J_{o}\right)+1
$$

subtracting 22 from 21 we have and rearranging the result we have

$$
V_{o c 2}=(k T / q) \ln \left(J_{s c 2} / J_{s c 1}\right)+V_{o c 1}
$$

After deriving the values of the photo current, and the open circuit voltage needed to solve the equation 4 considering the effects of texturing .Therefore, the most effective factors are the groove angle, and the angle of incidence, as shown in fig. 2, both variants must be considered in any treatment of texturing effects.
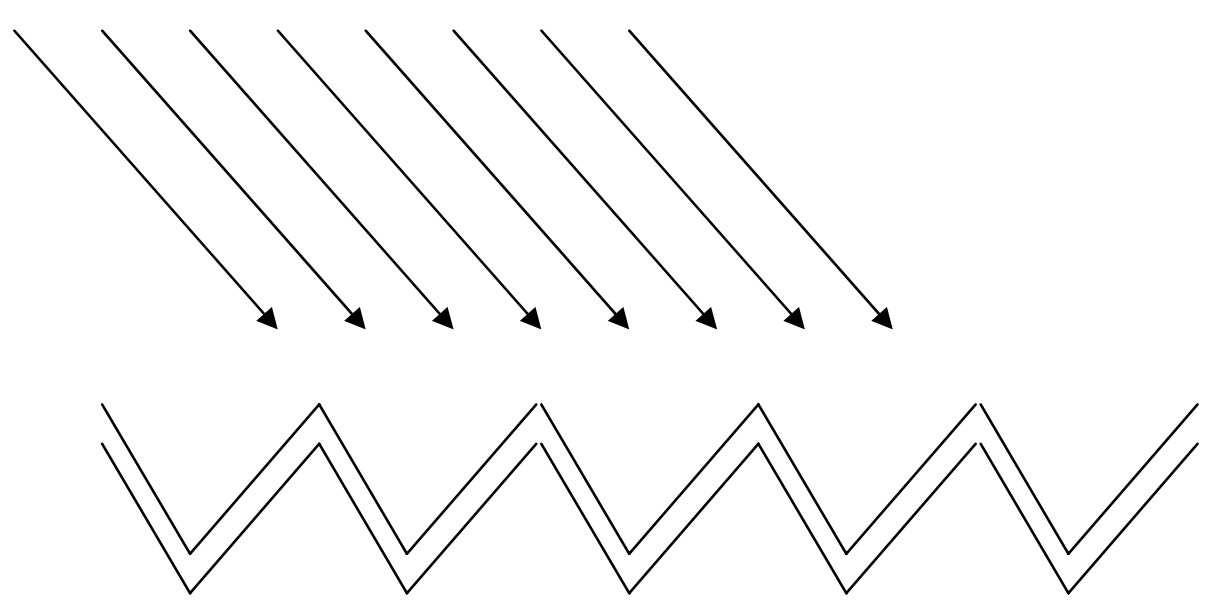

Fig. 2: The groove type of texturing and the incident light, it is clear that the two effects of groove angle and the angle of incidence on the light energy absorbed by the thin film layer 
When light is incident on the textured surface of the panel where we consider each groove as forming one cell. For certain groove angle $(2 \alpha)$ the quantity of light energy entering the cell depends on the angle of incidence (i) as shown in fig. 3, thus for angles of incidence greater than 2 only part of the groove of length $\mathrm{x}$ of the total length of the groove edge (E). From the geometry shown in fig.3.

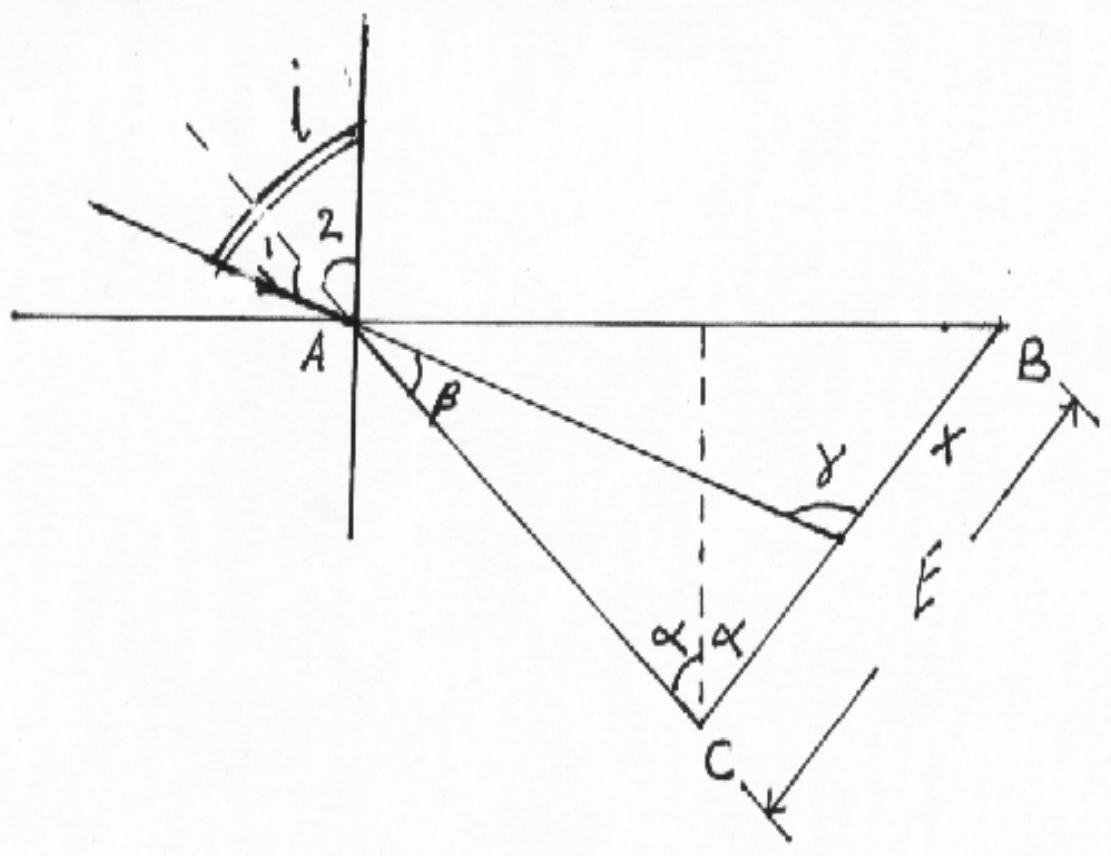

Fig. 3 : Shows the geometry of the incidence of light for certain angle (i) from which the illuminated part of the edge can be calculated and using some of the trigonometric relations for the triangle

$$
\begin{aligned}
& \angle i=\angle 1+\angle 2 ; \quad \angle \alpha=\angle 2 ; \angle \beta=\angle 1 ; \angle \gamma=\angle \beta+2 \angle \alpha \ldots \ldots \\
& \sin \alpha=\frac{O B}{C B} \quad ; \quad O B=E \sin \alpha \quad ; \quad A B=2 E \sin \alpha
\end{aligned}
$$

in order to find the length of the illuminated part $\mathrm{x}$ we use the law of cosines

$$
\frac{A B}{\sin \gamma}=\frac{x}{\sin (90-i)}
$$

using equations 24 and 25 and substituting in 26 we have

$$
\frac{x}{\cos i}=\frac{2 E \sin \alpha}{\sin (i+\alpha)}
$$


in the case of direct incidence of the light rays inside the groove, it can reflect all or part of the incident rays as shown in fig. 4 .

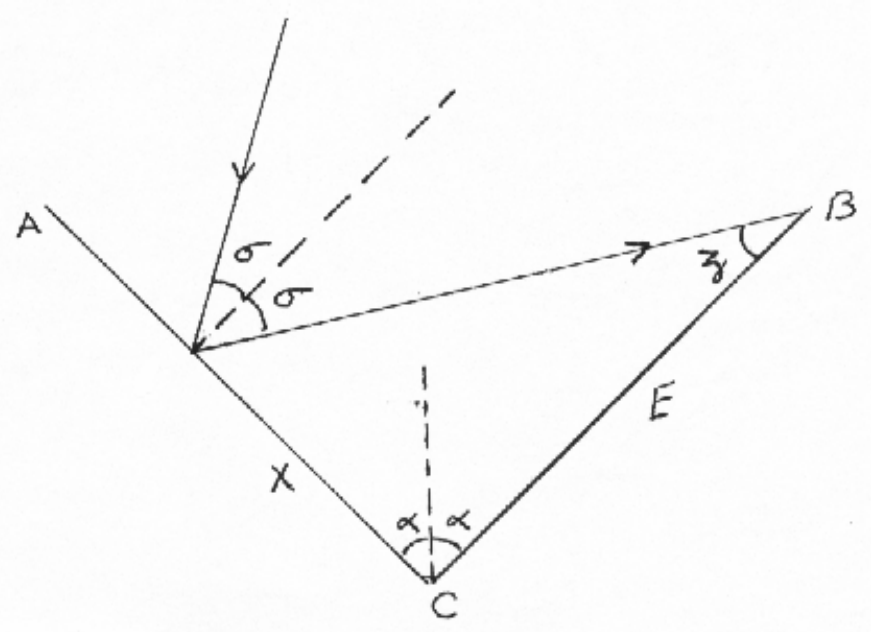

Fig. 4 : Geometry of the direct incidence of the light rays from which we can calculate the part from the edge ( $\mathrm{x}$ ) which can be incident on the edge CB from the edge AC

We can evaluate the effect of grooves in trapping the light rays by study of the geometry in fig. 4 in which a light ray is incident on the edge AC the part of the edge $\mathrm{x}$ which can reflect to the edge $\mathrm{CB}$ can be calculated in the following way :

$$
\sigma+\alpha=90 \quad ; \gamma=90-\sigma ; \quad \zeta+2 \alpha+\gamma=180 ; \quad \xi=90-2 \alpha+\sigma
$$

using the law of sine we have

$$
\frac{x}{\sin \zeta}=\frac{E}{\sin \sigma}
$$

from which we can see the effect of grooves in collecting light by secondary reflection . In order to fully consider the effect of texturing in collecting light rays, it must be considered for the two sides of the groove as shown in both equations 27 and 28 are used to find the quantity of the light collected by the groove. 


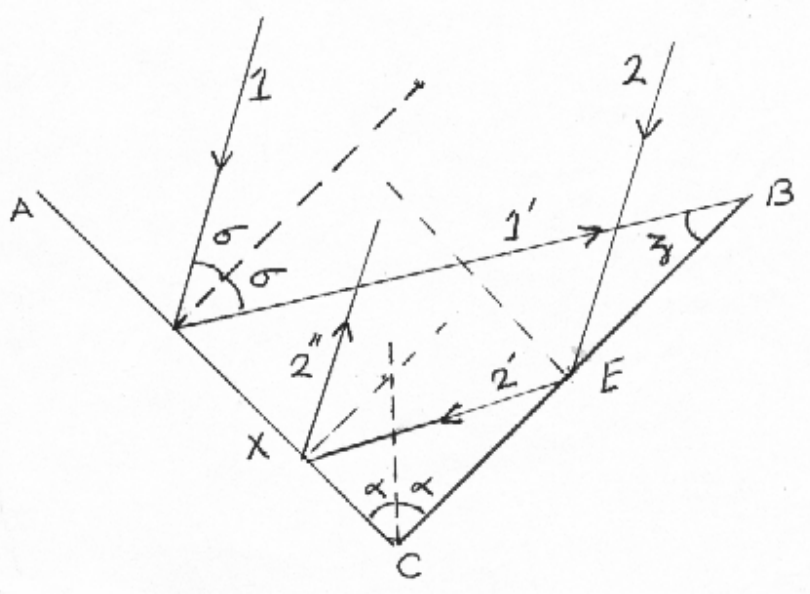

Fig.5 : The geometry of the incidence light on the two sides of groove rays 1 and 2 , for angle of groove equal $2 \alpha$

\section{RESULTS AND DISCUSSION}

In the theoretical part a method was proposed to study the effect of texturing (by using the idea of the grooves) on the efficiency of the solar panel for the (a-Si ) solar cells. The effect of the groove depend on two factors the angle of the groove and the angle of incidence of the light rays. Thus the cases of interest was studied for the angle of the groove ( the acute, the right and the obtuse angles), for each one of the former cases different angles of incidence was used a long calculation of the effect was made depending on finding the $\mathrm{I}-\mathrm{V}$ characteristics for each case depending on the equations we found in the former part. We consider the two side of the groove as representing a unit solar cell which repeated with the total number of the panel, as shown in fig. 2 .

The efficiency of the solar cell is constant, but as we will see from the following that this efficiency will be changed by the angle of incidence and by the angle of the groove. For this reason we will call this efficiency as the effective efficiency. The calculations depends on calculating the light energy that falls on the sides of the groove and reflected by other side and this depends on the angle of incidence and the angle of the groove. This is important because the incident rays from the sun always change its angle of incidence during the day. Thus the solar cell panel acts as a collector of the light energy and in the following, a clarifying of the angles of groove and incidence will be done for three cases of angles of the groove ( acute, right and obtuse) .

\section{Acute angles of the groove}

For the case of acute angles we take the angle 40 degrees as a representative of the case, Thus according to the equations derived in the theoretical part. The part of the incident energy and the reflected part from it is considered. Thus the quantity of the incident energy is found, therefore the main parameters of the solar cells are found ( the photo 
current, the short circuit current, the open circuit voltage). The drawing of the I-V characteristics is done for each angle of incidence. In fig. 6 the I-V characteristics for angle of the groove equal 40 degrees is shown

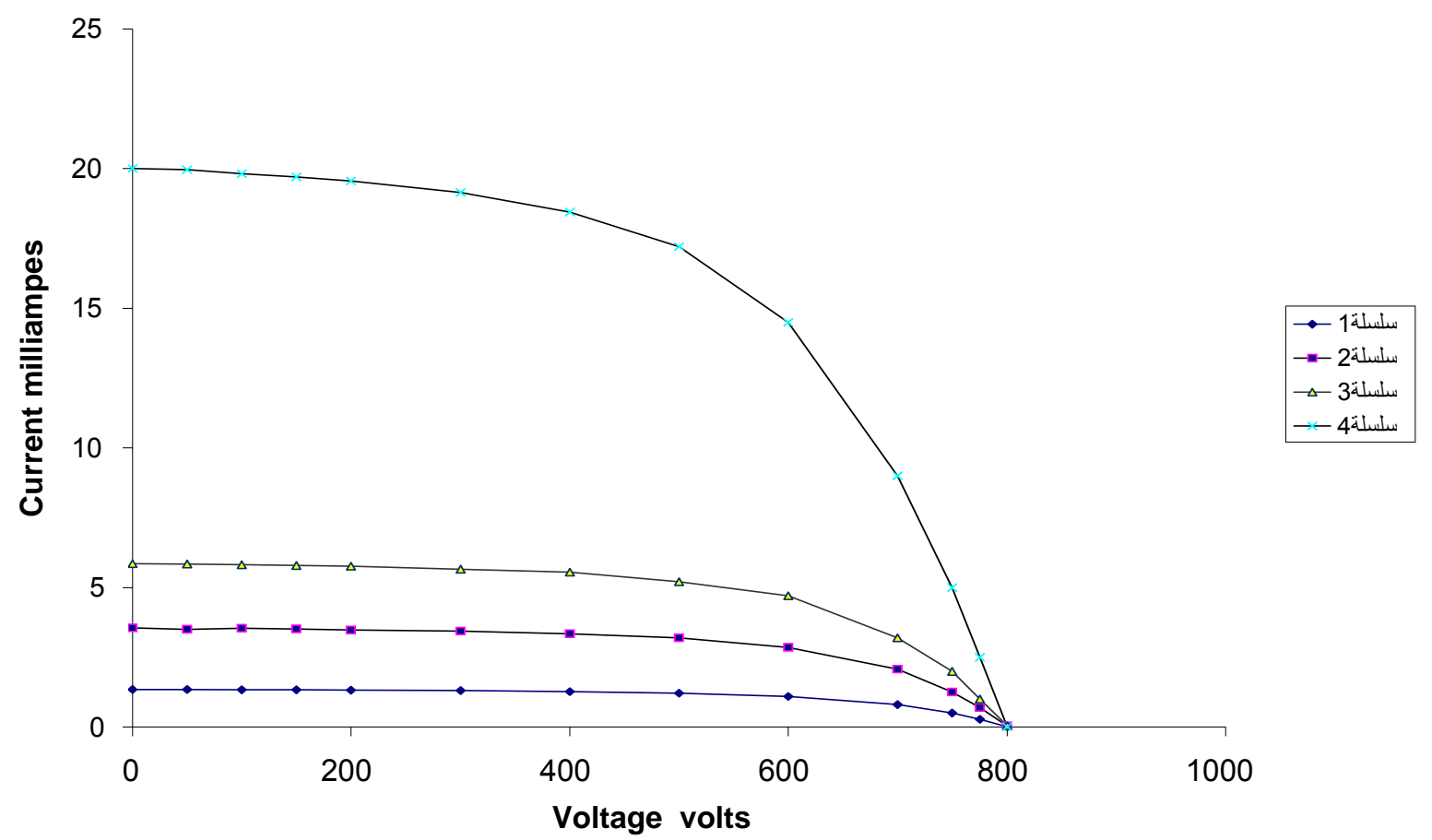

Fig. 6 : I-V characteristics for the amorphous silicon (a-Si) solar cells for groove angle 40 degrees and for different angles of incidence ( series 1,2,3 and 4 equals : 25,40 , 60 , and 90 degrees )

As shown in the figure the I-V characteristics are greatly affected by the change of the angle of incidence. For small angles of incidence the current in the cell is greatly diminished this due to the illumination of only a small part of one side of the groove. With increasing the angle of incidence an increase in the total current is noticed till the angle of incidence is greater than 70 degree then all the groove is illuminated by light and both sides of the groove is totally illuminated. And since the groove is narrow, so the reflected light from each side will be absorbed by the other side of the groove. The result of calculation using equation 28 shows that we have approximately total absorption of the incident light, so that the panel with this groove angle acts like a total absorber (black body). Thus as we see we have the highest current for the cell. This action continued up to the angle of incidence equal 110 (with respect to negative $\mathrm{X}$-axis according to which we count the angle of incidence) and then by the symmetry of the geometry of the groove will decrease again as for the left hand incidence (fig. 3,4 and 5 ).

From fig. 6 the efficiency of the solar groove for each angle of incidence is calculated by the change of efficiency with the change of the angle of incidence for the groove with angle 40 degree is shown in fig. 7 : 


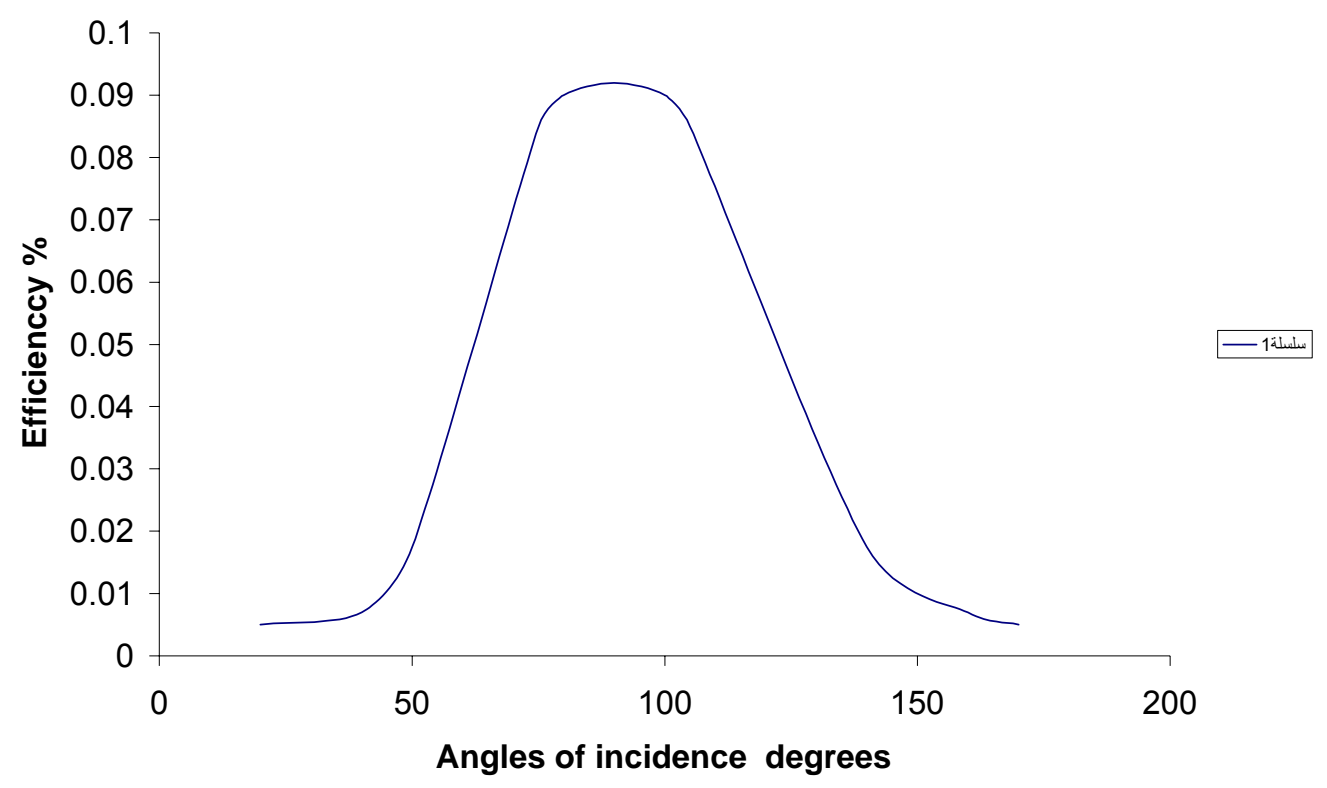

Fig. 7: The change of efficiency with the change of the angle of incidence for the groove with angle 40 degree is shown.

From the figure above a great value of efficiency is obtained for angles of incidence between 70 and 110 degrees for naked cell without any kind of reflection coating. But as it is evident this great efficiency is limited for limited range of angles of incidence. This case is of great importance for the case of having sun following technique, in this case we can obtain maximum efficiency for long time of the day.

\section{Right angle groove}

For the case of right angles we take the angle 90 degree as a representative of the case. Thus according to the equations derived in the theoretical part, the part of the incident energy and the reflected part from it is considered. Thus the quantity of the incident energy is found. The main parameters of the solar cells found (the photo current, the short circuit current, the open circuit voltage). The drawing of the I-V characteristics is done for each angle of incidence. In fig. 8 the I-V characteristics for angle of the groove equal 90 degree is shown 


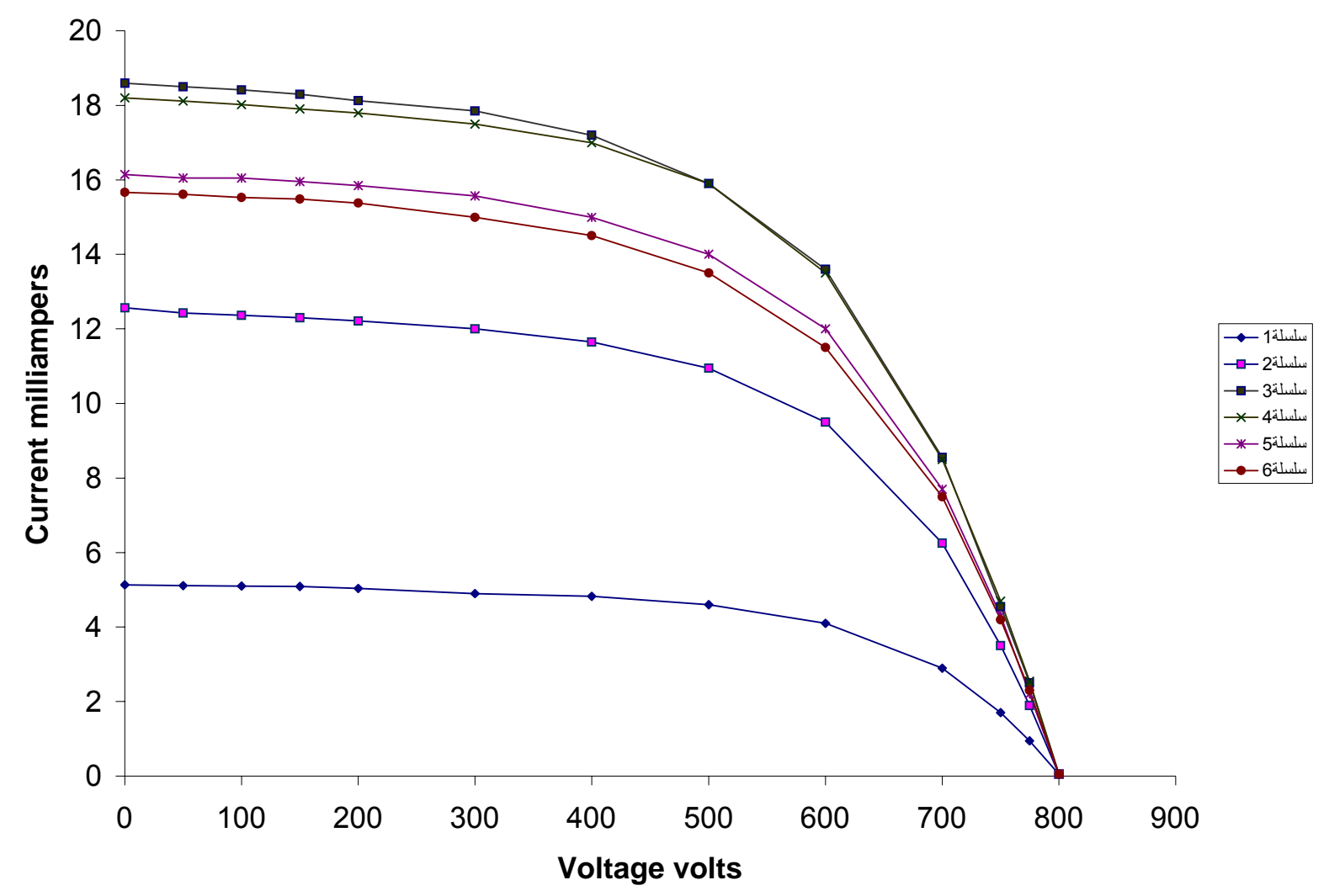

Fig.8: The I-V characteristics for angle of the groove equal 90 degree is shown by the curves from the lower one to the upper one are corresponding to angles of incidence equal to $(10,45,130,120,95$ and 85 degrees )

The characteristic feature of the fig. 8 in comparison with fig. 6 is the decrease of the effect of the angle of incidence( especially low angles ) in affecting the value of the current in the groove, also the change of the value of the current as evident from the figure. The angles of incidence 10 and 45 degrees represents rays from the left side of the groove, whereas the angles of incidence 130 and 120 degrees represents rays from the right left side of the groove. All the above incident rays are illuminating the groove partially so have low values of cell current. Also evidence from the figure that the angles of incidence 85 and 95 degrees represents rays incident on both sides of the groove but, illumination is total and so the reflected light from each side will be absorbed by the other side of the groove, and so we have the highest cell current . 
From fig. 8 the efficiency of the solar groove for each angle of incidence is calculated the change of efficiency by the change of the angle of incidence for the groove with angle 90 degree is shown in fig. 9 :

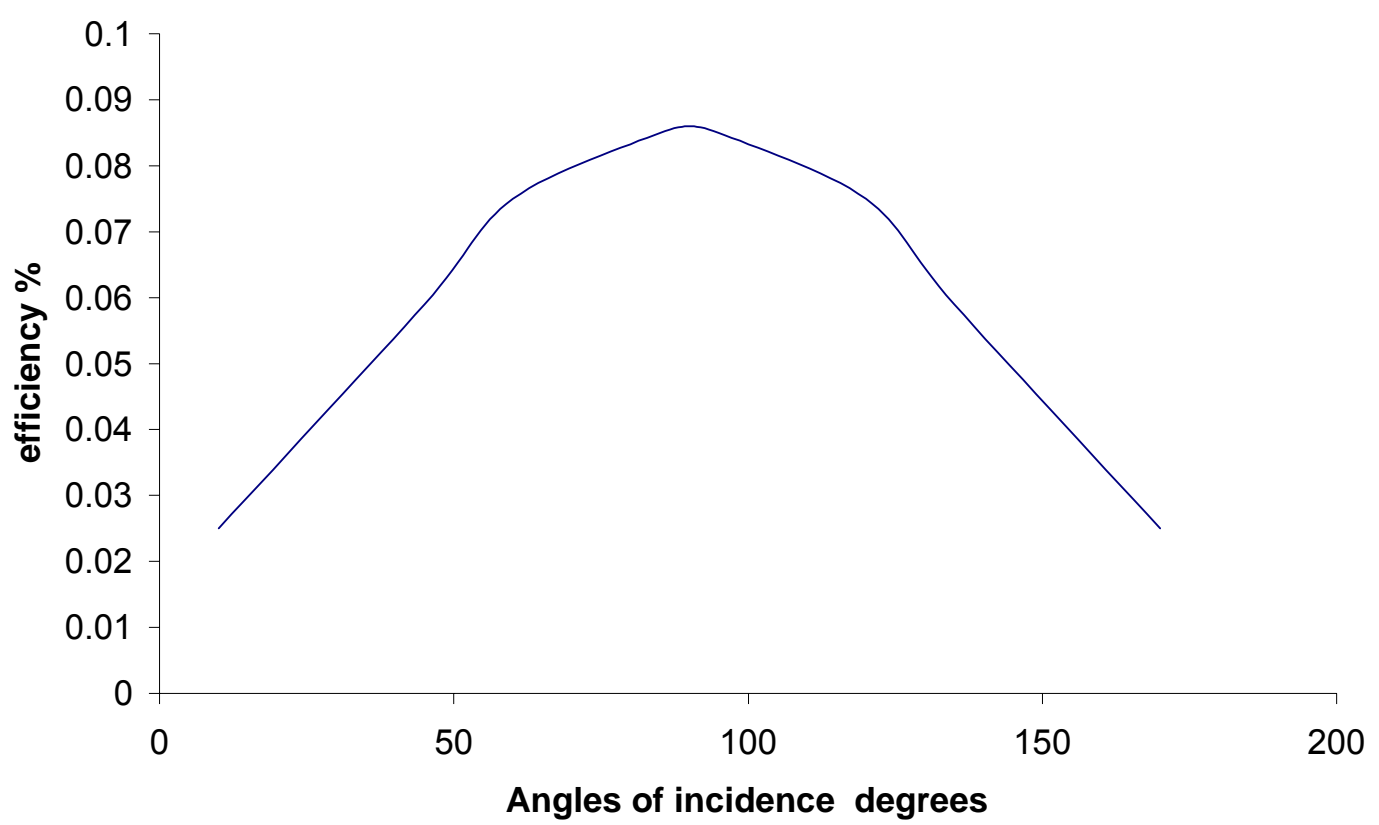

Fig. 9: The change of efficiency with the change of the angle of incidence for the groove with angle 90 degree is shown

From the figure above the largest value of efficiency $(0.085)$ is limited within a range for angles of incidence between 85 and 95 degrees for naked cell without any kind of reflection coating. But as it is evident this great efficiency is limited for limited range of angles of incidence. A lower efficiency (about $85 \%$ of the maximum ) covered a range about 70 degree. Also efficiency for low angles of incidence decrease less than for the case of the groove angle 40 degree, thus the ability of absorbing light is less.

\section{Obtuse angle groove}

For the case of obtuse angles we take the angle 110 degree as a representative of the case, the drawing of the I-V characteristics is done for each angle of incidence (the angles are 5, 20, 45, 65, 80 and 95 ) for each angle the reflections from both sides of the groove was calculated (to calculate the incident energy) because difference appear between the values of the sides then average of the two is found, using equations in the theoretical part, to calculate the I-V characteristics. In fig. 10 the I-V characteristics is shown 


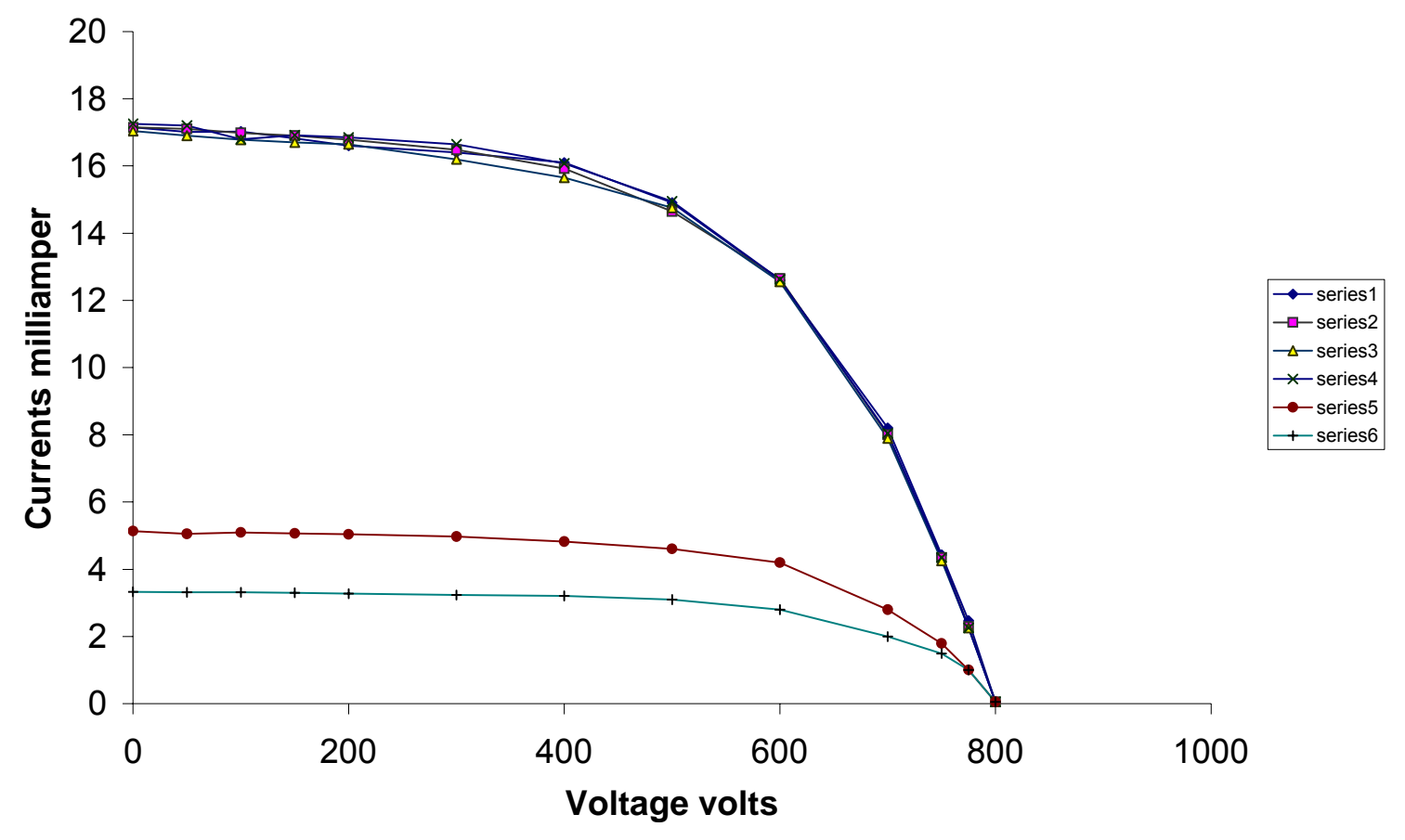

Fig. 10: The I-V characteristics for angle of the groove equal 110 degree is shown the series $(6,5,4,3,2,1)$ are corresponding to angles of incidence equal to $(5,20,45$, 65,80 and 95 ) degrees with respect to the negative $x$-axis (see fig. 3)

In comparing the characteristics of fig. 10 with those in fig. 6 and 8 , it is evident that their is a decrease of the effect of the angle of incidence (especially low angles) in affecting the value of the current in the groove. Also an abrupt change of the value of the current as the angles of incidence becomes 45 degree. When the incident rays are illuminating the groove. It is evident from the figure that for a wide range angles of incidence the values of cell current have near values from fig. 10 the efficiency of the solar groove for each angle of incidence calculates the change of efficiency with the angles of incidence is shown in fig. 11. 


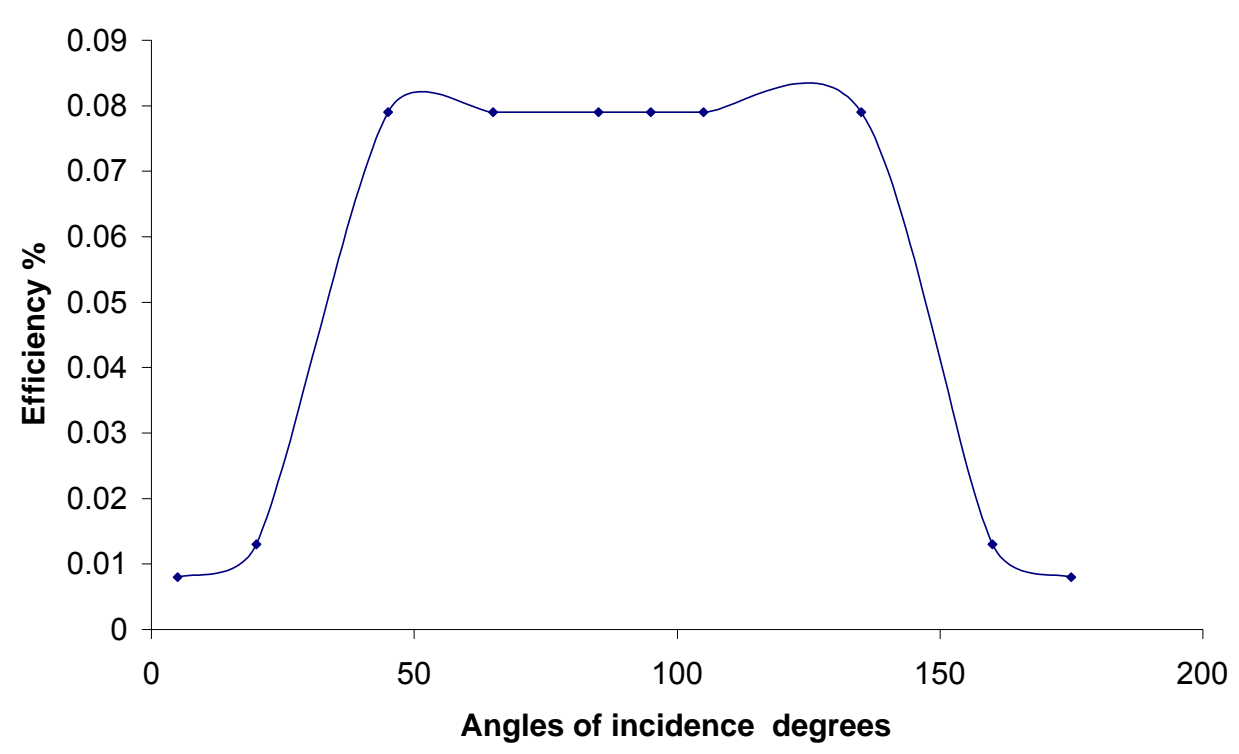

Fig. 11: The change of efficiency with the change of the angle of incidence for the groove with angle 110 degree is shown.

From the figure above the largest value of efficiency is highest (within a wide range of for angles of incidence from (50 up to 150 ) degree for naked cell without any kind of reflection coating. This enable this kind of texturing to be used for fixed panel systems.

\section{CONCLUSION}

1 - Texturing the solar cell surface by parallel grooves increase the efficiency by collecting the reflected light from one side of the groove by the other side of the amorphous silicon (a-Si ) solar cells.

2 - A theoretical method is found to calculate the effect of texturing on the efficiency of the amorphous silicon (a-Si ) solar cells whose surface have parallel grooves. A study of the effect of groove angles (acute, right and obtuse angles) and the angles of incidence from (0 up to 180) degree was done on the solar cell parameters (photo current, total current, short circuit current, open circuit voltage), by finding the amount of solar energy that is collected by the groove .

3 - For small groove acute angels ( 40 degree as example ) $100 \%$ collection of the incident energy is happened, this increase the efficiency, but this happens within limited range.

4 - Increasing the groove angles increases the range of maximum absorption but lowers efficiency by about $1 \%$. 


\section{REFERENCES}

Brendel, R.(1996)." Optical Design of Optical of Crystalline Thin Layer Silicon Solar Cells on Glass". Proc. 13th European Photovoltaic Solar Energy Conference, Nice , France.

Carlson, D. E. (1984). "Solar Cells, In Semiconductors and Semimetals", Vol. 21, Hydrogenated Amorphous Silicon Part D, edited by PJ. I. Pankove, Academic Press , New York.

Crandall, R. S. (1982). Transport in hydrogenated amorphous silicon $p-i-n$ solar cells, J. Appl. Phys . 53(4) figure.

Dragoman, D. ; Dragoman M. ( 2002)."Optical Characteristics of Solids", Springer , Berlin.

Faughnan, B. A. Moore ; Crandell, R. (1984). Relationship between collection length and diffusion in amorphous silicon, Appl. Phys. Lett. ,44 (6 ) , 613.

Green, M. A. (1995). " Silicon Solar Cells", Bridge Printing Ptg. Ltd.

Hovel, H. J.( 1975)." In Semiconductors and Semimetals". Vol.11, Solar Cell, edited by R . K. Willardson and A. C. Beer, Academic Press, New York.

Nelson, J.( 2003). "The Physics of Solar Cells, Properties of Semiconductor Materials", Imperial College, UK.

Swartz, G. A.( 1982). Computer model of amorphous silicon solar cell, J. Appl. Phys.,53(1), 712.

Swartz, G. A.( 1984)." Closed-Form Solution of I-V Characteristics for a - Si : H Solar Cells. In Semiconductors and Semimetals". Vol.21, Hydrogenated Amorphous silicon Part D, Edited by PJ. I. Pankove, Academic Press, New York.

Thorp, D. P. Campbell ; Wenham, S. R.( 1997). Absorption Enhancement in Conform ally Textured Thin-Film Silicon Solar Cells, http://www.pv.unsw.ed.au/. 